\title{
Downscaling climate change information: an essential ingredient to incorporate uncertainties into adaptation policies
}

\author{
$\underline{\text { B. Timbal }}^{\text {a }}$, Y. Wang ${ }^{\text {a }}$ and A. Evans ${ }^{\text {b }}$ \\ ${ }^{a}$ Centre for Australian Weather and Climate Research, Australian Bureau of Meteorology \\ ${ }^{b}$ South Australia Regional Office, Australian Bureau of Meteorology
}

Email: b.timbal@bom.gov.au

\begin{abstract}
:
A Statistical Downscaling Model (SDM) developed at the Bureau of Meteorology has now reached a stage where high resolution climate change projections of essential surface variables (rainfall and daily temperature), at a daily time scales, can be generated for the entire Australian continent. These projections are resolved onto on a horizontal grid of approximately 5-by-5km, and are based on an existing international database of global climate model projections used for assessment of climate change impacts as defined by the Intergovernmental Panel on Climate Change (IPCC).

The statistical linkage at the core of the SDM is based on the idea of daily meteorological analogues where optimal matching synoptic weather patterns are searched for in a historical database. The historical database employed here is made up of reanalyses of atmospheric circulation as observations for the large-scale predictors, and the Bureau of Meteorology's high quality in-situ observations consisting of a sparse network of about 100 to 200 stations across the continent. A combination of daily atmospheric variables are used for regional and seasonal optimization of the SDM (e.g. mean sea level pressure combined with an upper air moisture variable to predict rainfall or an upper level measure of temperature and air flow to predict surface temperatures) resulting in a total of 120 individual statistical models that describe a wide variety of Australian climates spanning from the tropical monsoon in the north to cool and temperate in the south. The 120 models are a product of:
\end{abstract}

- Regionalisation of the Australian continent into 10 climate zones where a different statistical model is optimized for each region;

- Meteorological analogues are chosen from within the same calendar season, thus 4 different models are optimized for each calendar season; and

- Analogues are searched separately for the three surface predictands (Rainfall, $\mathrm{T}_{\max }$ and $\mathrm{T}_{\min }$ ).

While the optimization and original application of the SDM was based on in-situ data, here we present the application of the technique to the latest surface gridded observations produced by the Bureau of Meteorology as part of the Australian Water Availability Project (AWAP). In this communication, we present and discuss the evaluation of the results using the gridded observations, including:

- The ability of the technique to reproduce the mean and variance of the observed local series;

- The ability of the technique to reproduce day-to-day variability, inter-annual variability and long-term trends; and

- The ability of the technique, despite being based on a univariate approach, to reproduce the observed relationships amongst individual predictands (rainfall and temperature).

Following this, each of the individual SDMs are applied to climate scenarios described by a suite of global climate models. The flexibility and low cost of the SDM makes for easy application to the large number of existing climate simulations, and to sample the uncertainties attached to the plausible future emission trajectories as well as the possible response of the climate system as modelled by the current climate models. In this regard, the SDM represents an essential ingredient for assessing future climate change uncertainties at a scale relevant for local climate impact studies. It has been noted in recent applications of downscaled climate projections presented here, that researchers can produce detailed climate change impact studies allowing for the development of well informed climate change adaptation policies.

Keywords: Statistical Downscaling Model (SDM), Gridded projections, Australian continent, 


\section{INTRODUCTION}

A cornerstone of scientific projections used to examine the possible impact of human induced climate change on any aspect of the society or the natural environment is the general circulation model (GCM). Essentially, a GCM solves sets of complex mathematical equations that describe atmospheric and oceanic circulation and interactions that govern the Earths climate system. These equations are solved on relatively coarse grids. Reducing the size of the grid cells is computationally expensive, particularly when simulations require long GCM integration to adequately simulate the slow response of the climate system to external anthropogenic forcings. In addition, the uncertainties attached to the simulation of the future climate (e.g. using different external forcings or using different configurations of the same GCM to test the sensitivity of the model to the external forcings), means a large number of simulations is preferable for evaluation purposes.

Therefore, there exists a trade-off between high grid resolutions desirable to inform climate change impact studies, the computing cost associated with these simulations, and the optimum modelling framework to estimate the uncertainties attached to these climate change projections. Currently, most GCMs are integrated with a horizontal resolution ranging from 100 to $300 \mathrm{~km}$. Although horizontal resolutions have been increasing over the last two decades (e.g. the $4^{\text {th }}$ IPCC assessment, Solomon et al. (2007) and the previous assessments), this increase is not commensurate with the overall increase of computing power. This can mostly be attributed to two reasons: 1 . GCMs are increasing in complexity and becoming more expensive to integrate and 2. A large part of the increased computing power has been dedicated to increasing the sampling of the uncertainties attached to the projections.

Climate change impact assessments often require point specific climate projections since local impacts are dependent on fine-scale climate variations. While it is possible to integrate a climate model with a very high horizontal resolution (e.g. very high climate simulations with a resolution as fine as $10 \mathrm{~km}$ have been completed), it does not provide the full gamut of information about the changing climate necessary to make meaningful risk management decisions. The importance of small scale information is particularly apparent in regions with complex topography, coastal locations, and in areas of highly heterogeneous land-cover. This highlights a gap between what is required to develop climate change adaptation policies and the climate change projections obtained from GCMs. Tools to downscale from the coarse information provided by GCMs to finer spatial scales are therefore required. In general, it is the spatial downscaling that is of interest here, temporal downscaling is not necessary since GCMs provide information at high frequency (less than hourly), although GCM data are rarely stored at frequency higher than daily time-scale.

One method to bridge the gap is to use a statistical downscaling model (SDM). SDMs are based on the premise that the regional climate is conditioned by two factors; the large scale climatic state and local physiographic features. From this perspective, regional or local climate information is derived by first determining a statistical model which relates large-scale climate variables (or predictors) to regional and local variables (or predictands). The large-scale outputs from GCM simulations are then fed into the SDM to estimate the corresponding local and regional climate characteristics for both current and future climates. The Australian Bureau of Meteorology (BoM) has developed a SDM using the idea of meteorological analogues (Timbal and McAvaney, 2001). This is one example of a more general type of technique based on weather classification methods in which predictands are chosen by matching previous (i.e. analogous situations) to the current weather-state. This type of SDM was first developed for generating daily maximum and minimum temperatures $\left(T_{\min }\right.$ and $\left.T_{\max }\right)$ and was subsequently extended to rainfall occurrences (Timbal et al., 2003) and then to rainfall amounts (Timbal, 2004). The technique was then applied to other regions across the Australian continent to include tropical and extra-tropical climate regimes (Timbal et al., 2008).

Typically, a SDM is trained on data recorded at observing stations such that climate change projections for the same location can be provided. This is the case for the existing SDM, which deliver projections across Australia for Rainfall, $\mathrm{T}_{\max }$ and $\mathrm{T}_{\min }$ (Timbal et al., 2009). The ability of SDMs to provide station based information is often regarded as an advantage compared to dynamical downscaling techniques. However, many impact studies require high resolution gridded information and often several predictands simultaneously on the same grid. One possibility is to use the same type of climate model but on limited area. A regional climate model (RCM), forced at its boundaries by conditions set by GCMs, can be pushed to resolutions down to tens of kilometres at reasonable computing cost, e.g. the Climate future for Tasmania project, Grose et al. (2010). While this a feasible option, RCMs remain costly if the uncertainties attached to the projections is to be fully evaluated through acquisition of several integrations. 
Here we present as an alternative to RCMs, an extension of the SDM to gridded observations. This offers a cost effective way to deliver high resolution downscaled projections on a regular grid (5km resolution) and for three predictands: Rainfall, $\mathrm{T}_{\max }$ and $\mathrm{T}_{\min }$.

In this paper, we describe how the SDM was applied to gridded observations (section 2), present the accuracy of the model gridded outputs (section 3), evaluate the ability of the model to produce realistic relationships amongst the predictands (section 4) and illustrate the existing downscaled climate projections across the Australian continent based on available GCMs (section 5). Some final remarks are proposed in section 6.

\section{DATA AND METHODS}

The SDM was originally developed and validated on high quality (HQ) station data (Timbal et al., 2009) and is easily applicable to other predictands series within the same area. In addition, the versatility of the SDM has allowed it to be readily applied it to the $5 \mathrm{~km}$ gridded analyses generated by the Bureau of Meteorology as part of the Australian Water Availability Project (AWAP) (Jones et al., 2009). The AWAP analysis includes precipitation, maximum and minimum temperature, obtained by interpolating surface measurements onto a $0.05^{\circ}$ grid (roughly equivalent to $5 \mathrm{~km}$ by $5 \mathrm{~km}$ resolution). Here we make use of AWAP data from 1958 to 2006 as our database to search for analogue situations. Ten distinct climate regions loosely based on rotated empirical orthogonal functions of annual rainfall were used to apply the SDM across the entire Australian continent (Timbal et al., 2008). The same regions were used but the boundaries between them were drawn as straight line segments between regions unless orographic features such as the Great Dividing Range across Eastern Australia were found to be meaningful (Fig. 1). Using the same choice of analogues, the predictands from the HQ stations were replaced by

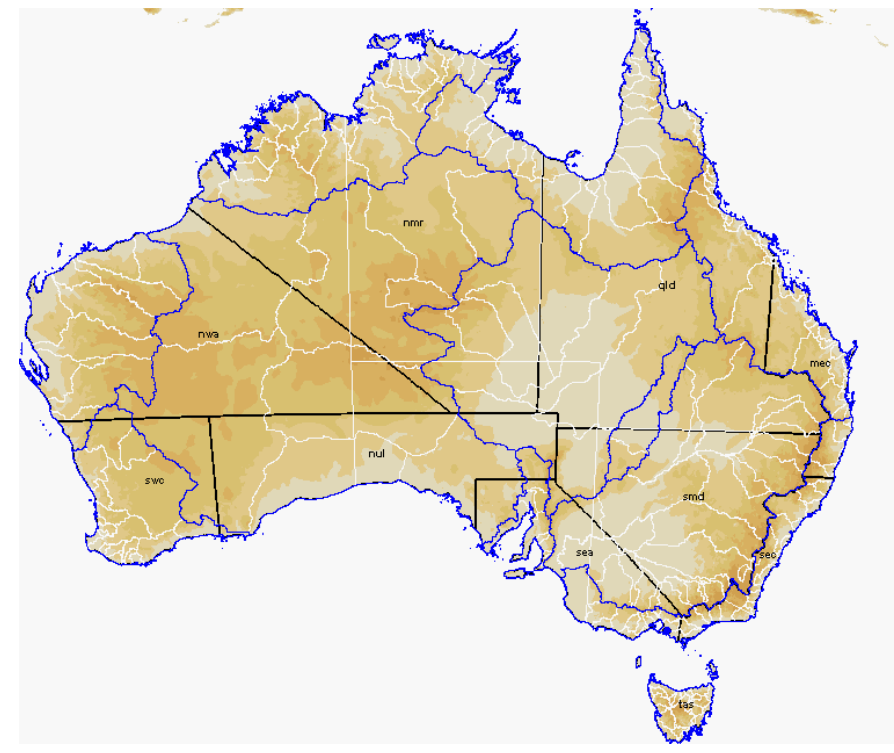

Figure 1. Australian continent, including major (blue lines) and minor (white lines) catchment boundaries; colored shading shows the orography (darker color for higher elevations) superimposed with the 10 climate regions used bv the SDM.

those from the gridded AWAP data. This made the application of the SDM relatively trivial and the gridded projections were generated without specific optimization.

Daily large-scale atmospheric "predictors" were obtained from the global $2.5^{\circ}$ re-analyses of the atmosphere completed jointly by the National Center for Environmental Prediction (NCEP) and the National Center for Atmospheric Research (NCAR), labelled NNR (Kalnay et al., 1996).

Once validated, the SDM was applied to GCMs from the Coupled Model Intercomparison Project $\mathrm{N}^{\circ} 3$ (CMIP3) assembled as part of the IPCC $4^{\text {th }}$ Assessment (Solomon et al., 2007). Since the resolution of GCMs varies, each was interpolated back onto the NNR $2.5^{\circ}$ grid. To ensure the best possible sampling of the uncertainties attached to the future projections due to model sensitivities, all GCMs were considered. Multiple daily fields are required to perform the statistical downscaling however, and only 11 out of 23 GCMs included in CMIP3 had stored all the required data. Another limitation arises from the fact that in CMIP3, daily fields were only requested for three "time-slices": 40 years from 1961 to 2000 representing the current climate and two 20 years future periods from 2046 to 2065 and from 2081 to 2100 . These time-slices are currently the only periods for which it is possible to obtain downscaled climate change projections with the SDM or any forms of downscaling relying on daily data as input. It is worth noting that this is far from ideal since a short 20-year period will have a decadal variability component which is difficult to separate from the climate change "signal". In this regard it is important to use as many GCMs as possible, as each 
individual model contributes to sampling decadal variability alongside the model sensitivity to the external forcing. In order to sample the uncertainties associated with future projections due to different possible future anthropogenic emissions of greenhouses gases, two emission scenarios are considered (out of the 6 recommended by the IPCC): A2, a high emission scenario, and B1, a low emission scenario.
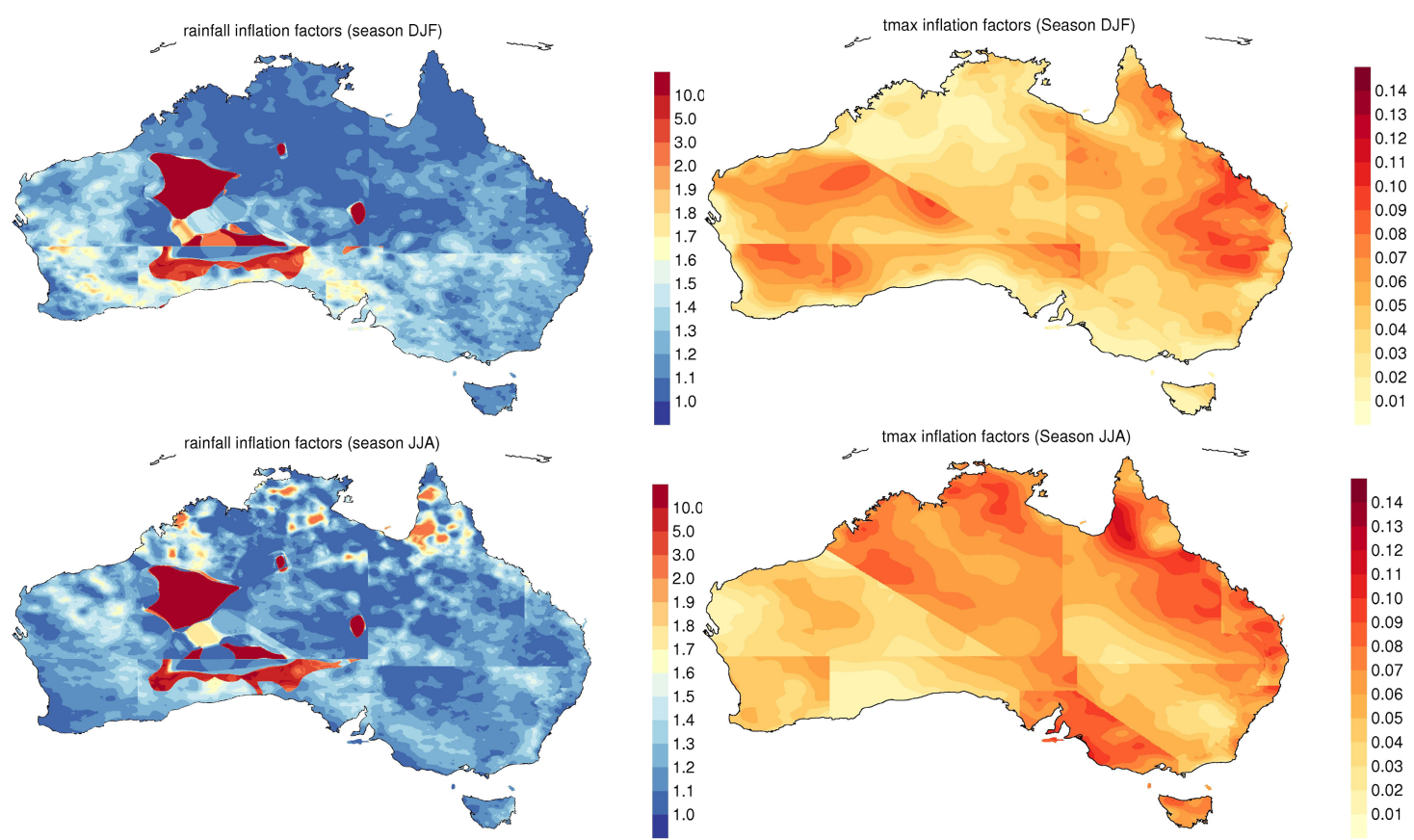

Figure 2. Inflation factors for rainfall (left) and $\mathrm{T}_{\max }$ (right) in summer (upper row) and winter (lower row). [NB: the high values in the middle of the continent for rainfall (in red) are due to missing AWAP data for which in fact no rainfall projection are possible and thus should be ignored].

The analogue method, as any statistical downscaling method, has a tendency to underestimate the observed variance (von Storch, 1999). This is particularly critical for rainfall. Examination of the rainfall probability distribution functions (PDFs) drawn from the downscaled series shows that extreme events are not adequately captured and that, instead, the SDM reproduces a higher frequency of low rainfall events. Therefore for rainfall, the variance underestimation leads to a dry bias. A simple inflation factor was developed for daily rainfall (Timbal et al., 2006) and applied to HQ stations (Timbal et al., 2009). This approach has since been further developed by Evans et al. (2011) for both rainfall and temperature on a regional and seasonal basis. The factor is based on the observation that the daily rainfall PDF is best approximated by a gamma distribution function (Wilks, 2006).

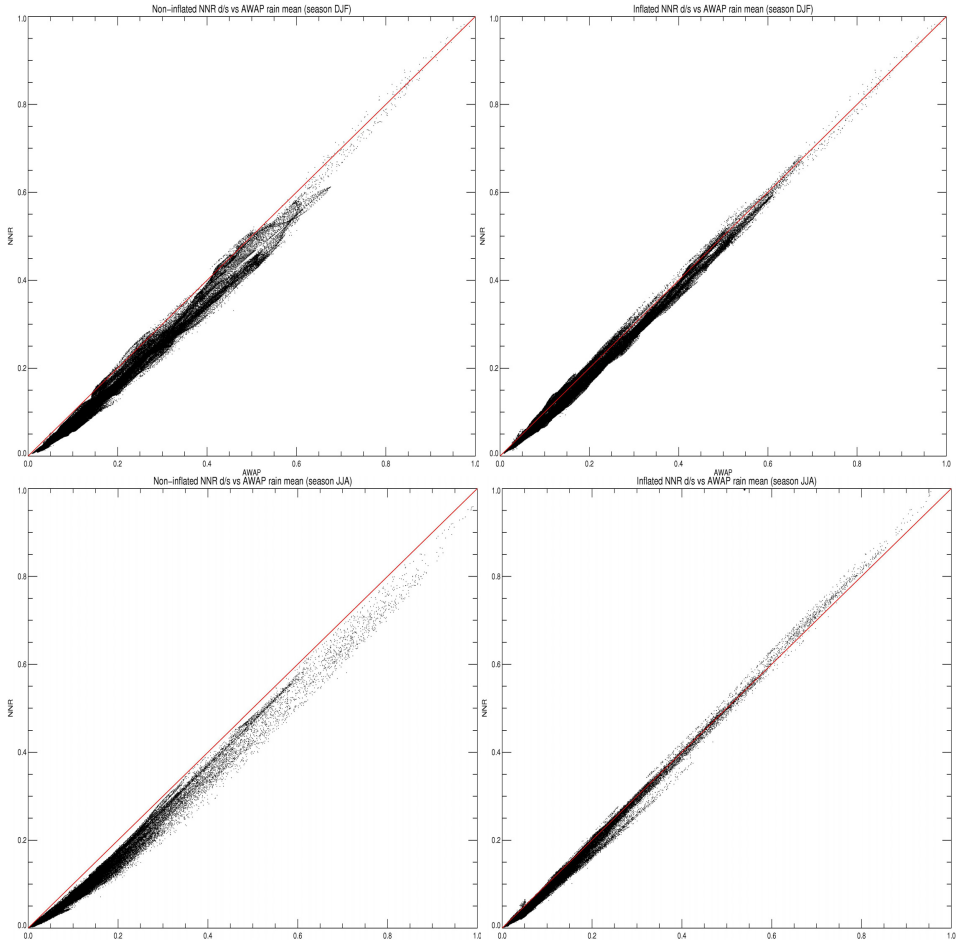

Figure 3. Scatter plots of mean rainfall for summer (upper row) and winter (lower row) without (left) and with (right) the inflation factor. 
Using the shape and scale parameters describing the gamma PDF, an inflation factor was computed at each and every point on the AWAP gridded surface. Figure 2 (left panels) shows inflation factors for summer and winter rainfall mapped across the Australian continent. These maps are indicative of the performance of the SDM. Higher factors indicate that the downscaled variance is underestimated more than that in regions with lower factors (typically in regions and seasons with few rainfall occurrences: i.e. in the North in winter and in the South in summer).

The impact of the inflation factor on the NNR based downscaled reconstruction of the AWAP rainfall can be seen on the mean (Figure 3) and on the

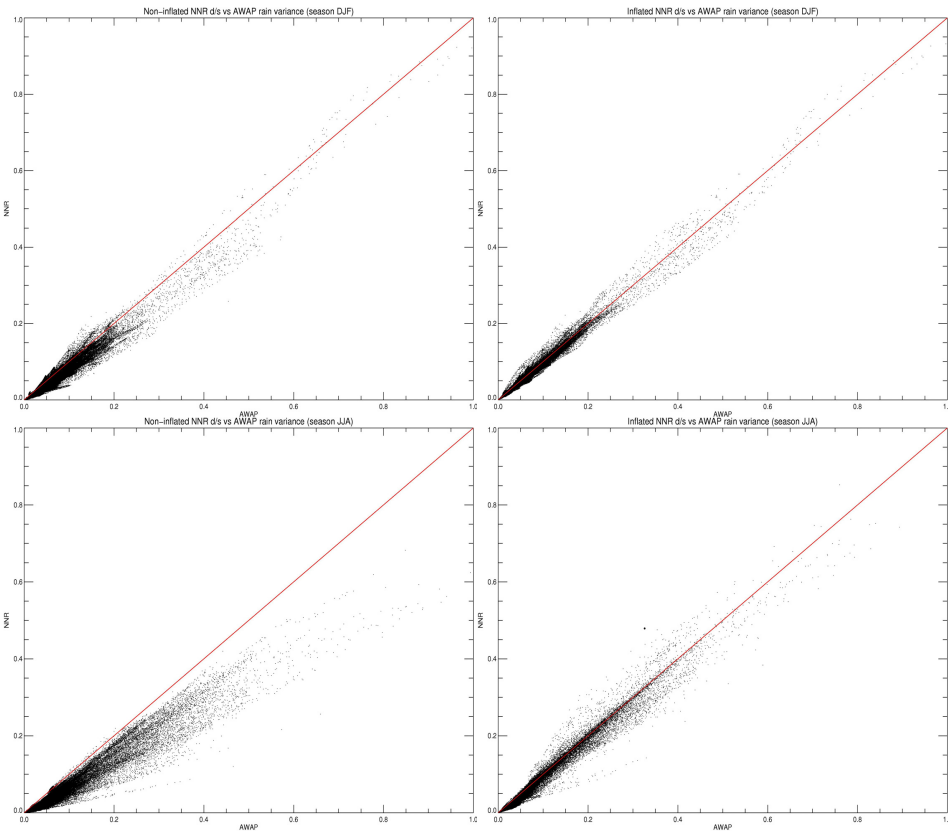

Figure 4. Scatter plots of rainfall daily variance for summer (upper row) and winter (lower row) for the observed versus reconstructed series, with (right column) and without (left column) the inflation factor

daily variance (Figure 4) for all points of the AWAP grid in summer and winter. Removing the systematic underestimation of the variance, the inflation factors have eliminated the dry bias evident in the mean rainfall.

For temperature, a similar procedure was followed; except that the comparison of the PDF was made with the theoretical Gaussian distribution. The temperature inflation factors adjust the values of each of the analogue temperatures relative to its distance from the observed mean. An additive term is applied to each downscaled day, the value of which is determined by the correction parameters (Evans et al., 2011) which are mapped in Figure 2 (right panels). As with rainfall, these panels reveal the performance of the SDM at reproducing temperature variability over regions for a given season. In most instances, the required inflation factors are relatively
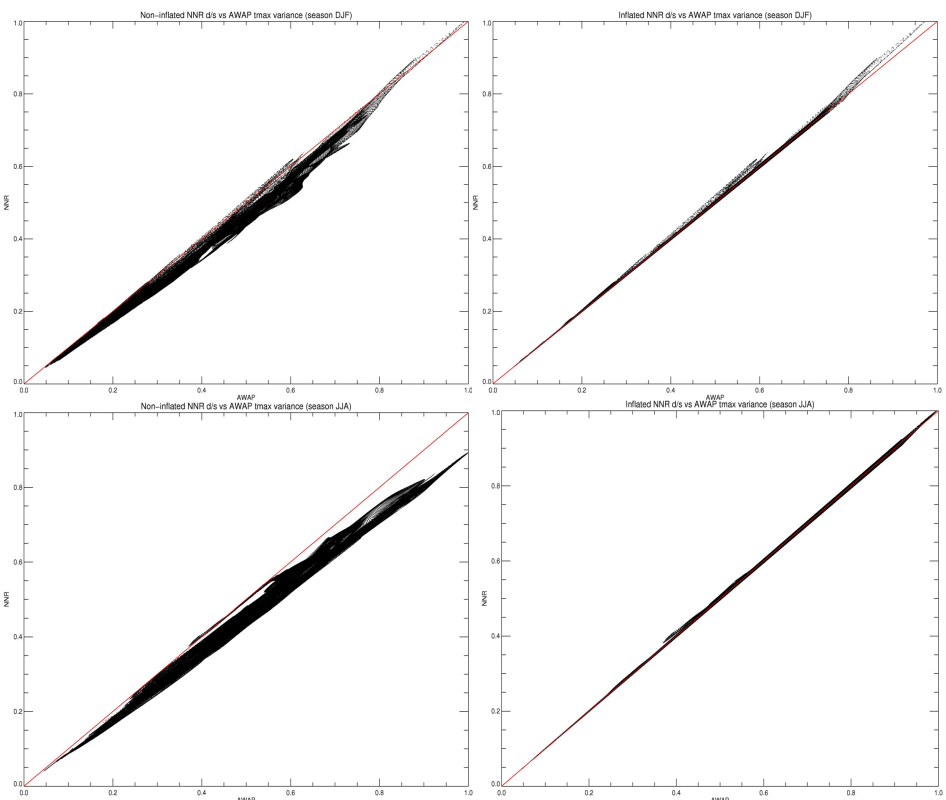

Figure 5. As per Figure 4 but for $T_{\text {max }}$ small for both $\mathrm{T}_{\max }$ (Figure 2) and $\mathrm{T}_{\min }$ (not shown). The effect of the inflation factors on reconstructed variances is clear (Figure 5) as the variance of the series for each grid point closely matches the AWAP observation. This correction has no particular effect on the mean of the series since the temperatures were not biased to start with. In addition, the methodology employed here for the correction of downscaled rainfall and temperature variance has a negligible effect on the root mean-square error of the downscaled series but does lead to only a very small improvement in the inter-annual variance and long-term trends captured by the SDM (Evans et al., 2011). 


\section{EVALUATION OF INDIVIDUAL GRIDDED PREDICTANDS}

One of the key statistics for any downscaling technique is the ability to reproduce the correct daily values. We computed the correlation between the observed and reconstructed series at the daily time-scale for all three predictands and all seasons (illustrated in Figure 6 for summer and winter for both rainfall and $\mathrm{T}_{\max }$ ). Overall the technique performed as well with the gridded observations as it did with the HQ network (Timbal et al., 2009) despite not being specifically fitted to this particular dataset. Correlations vary spatially and seasonal, and for rainfall range from 0.3 to 0.6 , and between 0.6 and 0.8 for temperature with.
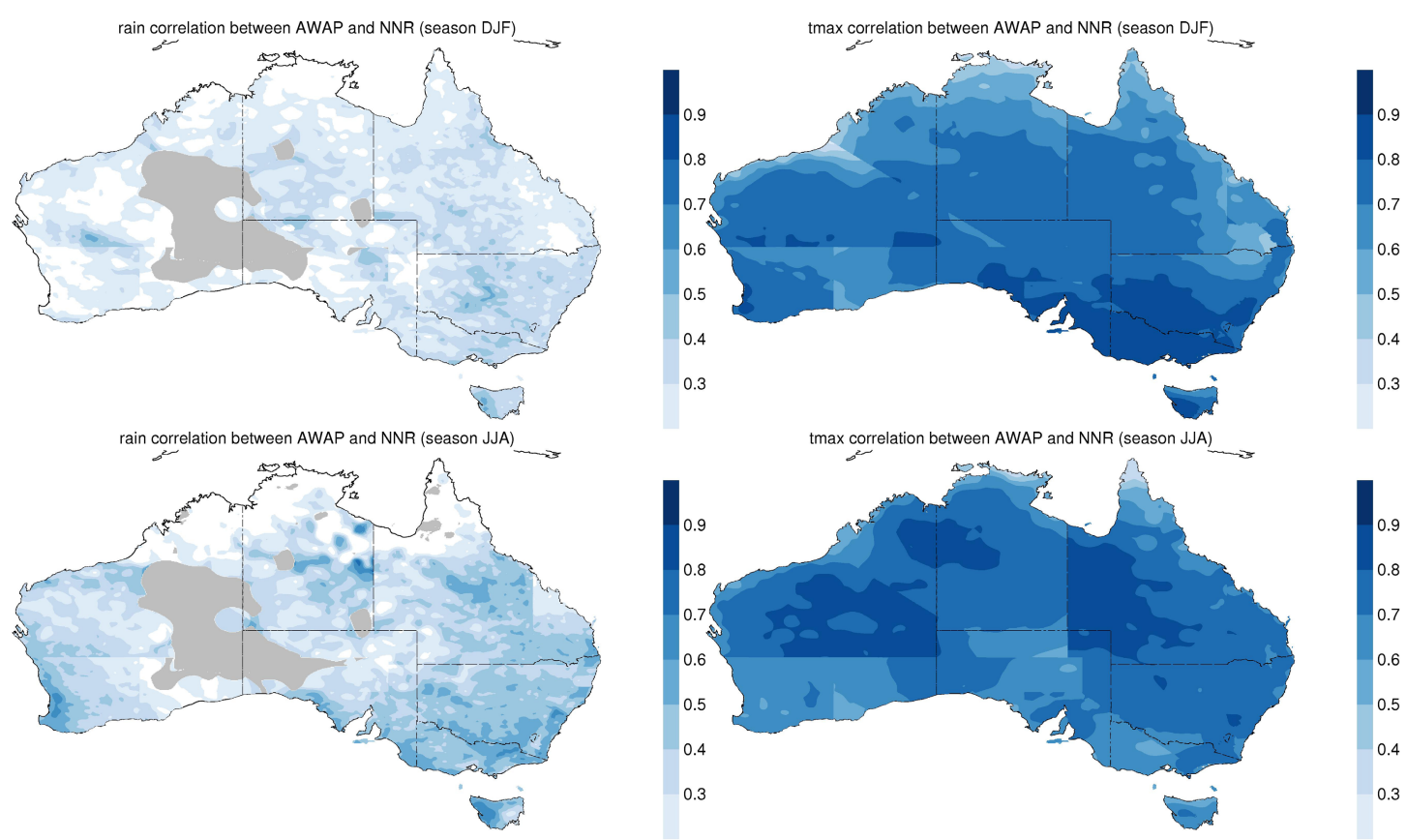

Figure 6. Maps of correlation coefficients between daily observed and reconstructed series for rainfall (left column) and $\mathrm{T}_{\max }$ (right column); for summer (upper row) and winter (lower row).
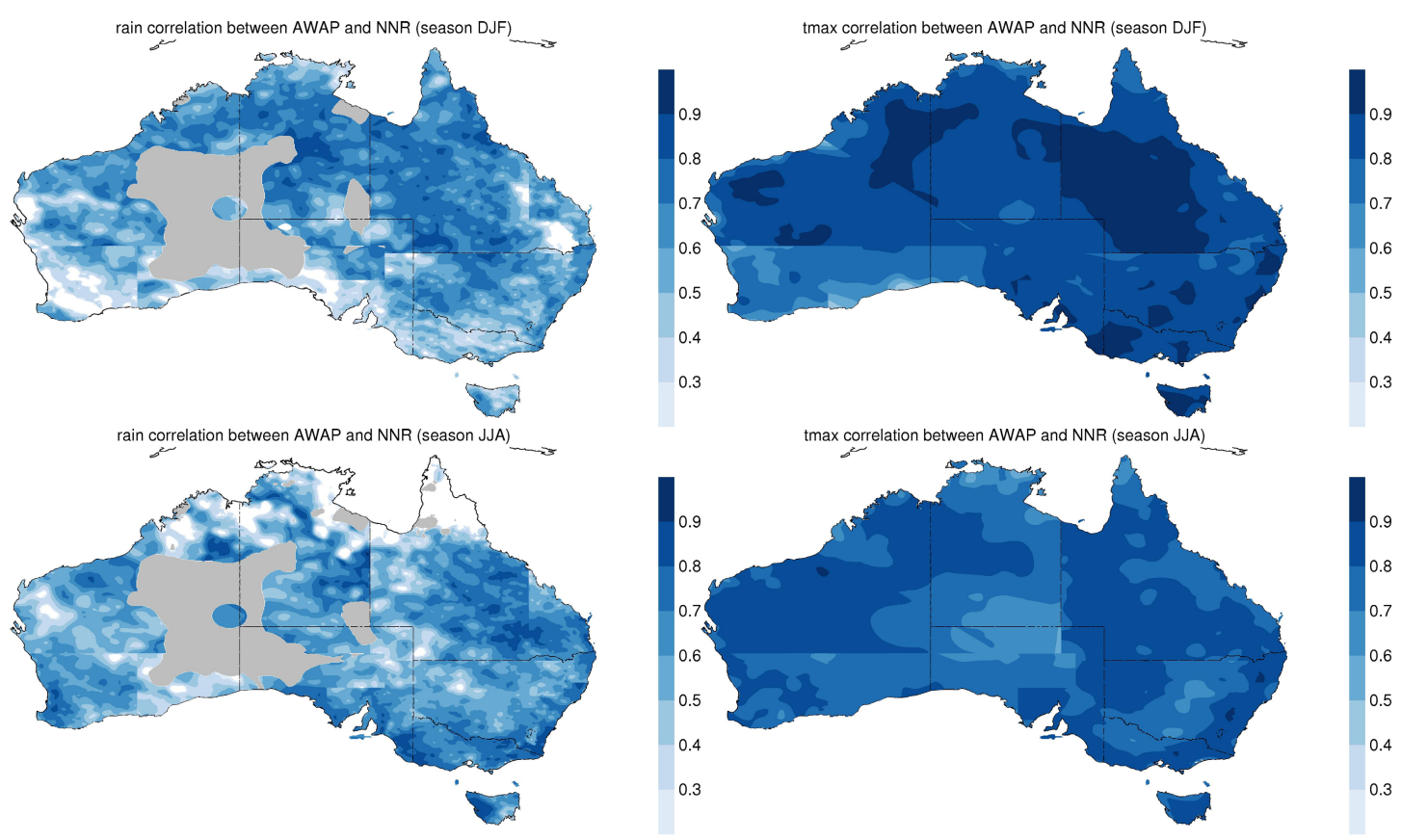

Figure 7. As per Figure 6 but for the correlation coefficients of seasonal mean. 
Overall, correlation coefficients for rainfall are lower and less coherent, reflecting the effect of local influences not necessarily controlled by the large-scale predictors as assumed by the SDM. Correlations are particularly low for the Tropics where the extension of the technique is problematic and that only large-scale rainfall was found to be the only suitable predictor (Timbal et al., 2008).

In addition to the reproduction of day-to-day variability, in the context of the application to a changing climate, the ability to reproduce year-to-year variability and long-term trends is essential. The inter-annual variability of the downscaled series is well correlated with the observed series for all predictands (shown in Figure 7 for summer and winter for both rainfall and $\mathrm{T}_{\max }$ ) with most areas showing correlations between 0.5 and 0.9. Interestingly, in some instances, the inter-annual variability of the seasonal means is well reproduced even when daily correlation is low (e.g. for rainfall in the Tropics in summer).
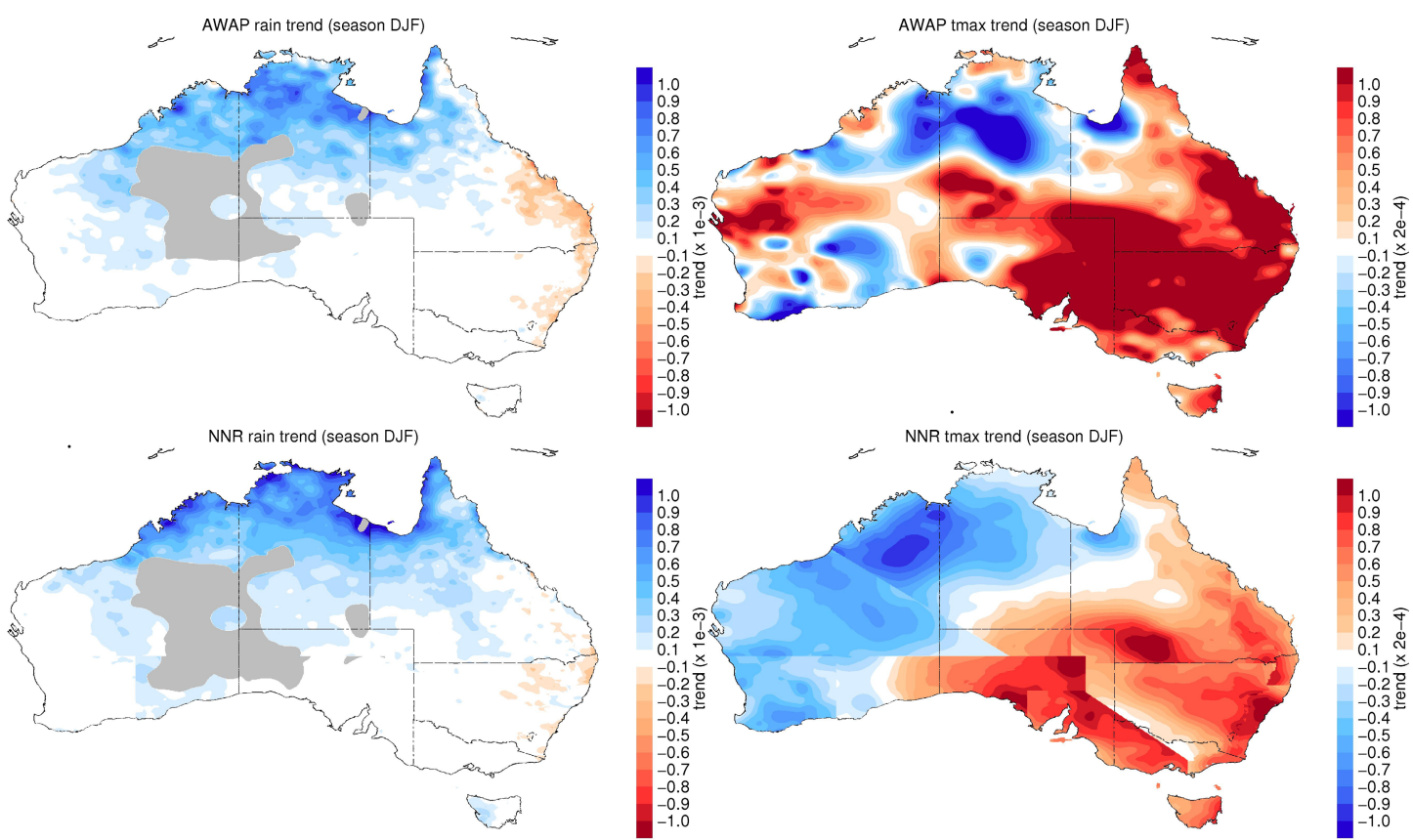

Figure 8. Long-term (1958 to 2006) linear trends for rainfall (left column) and $T_{\max }$ (right column) observed (upper row) and from the NNR-based downscaled reproduction of the observations (lower row).

Finally the technique's ability to reproduce long-term trends was also evaluated (shown in Figure 8 for summer for both rainfall and $\mathrm{T}_{\max }$ ). Overall it was found to be satisfactory since the SDM is able to capture the sign and spatial patterns of observed trends (e.g. summer cases for $\mathrm{T}_{\max }$ and rainfall). The magnitude is realistic, particularly for rainfall. In the case of temperature, it was found that, in general the technique underestimates the long-term trends. This is likely to be due to an under-reproduction of the magnitude of the inter-annual variability as noted in Timbal et al. (2008) for the HQ temperature stations when no inflation factor was used. Evans et al. (2011) noted that the newly developed inflation factor for temperature had only a limited positive effect in improving the reproduction of the observed magnitude of the inter-annual variability and long-term trends.

\section{MULTIVARIATE EVALUATION}

The application of the AWAP gridded products has allowed users to access several variables (rainfall, maximum and minimum temperature) on a same grid. While the downscaled variables are independently derived they need to be physically consistent to be usable in impact modelling. Therefore it is necessary to conduct a multivariate evaluation of the SDM. We test that the relationships between the downscaled predictands are consistent with known relationships (Power et al. 1998).

While the methodology employed here is based on a univariate approach (i.e. analogue days are chosen from different individual SDMs and are in most cases from different days), the results are very encouraging, suggesting that the methodology chosen is not an issue. Figure 9 shows the reproduction of the relationship 
between rainfall and the mean of $\mathrm{T}_{\max }$ and $\mathrm{T}_{\min }$ for both summer and winter. This is a very useful comparison involving all three predictands generated and indicates that the SDM captures the direction and strength of the relationship between these variables. There are areas and seasons where the relationship is not well captured, but in most instances the poor reproduction of the relationship between the three predictands can be traced back to one of the predictands not being well represented: e.g. the south-west corner of Western Australia in summer, where the predominantly negative relationship between rainfall and $T_{\text {mean }}$ is not reproduced; that region was noted earlier as an area where correlation for daily rainfall was fairly low (bottom left panel in Figure 6). Other relationships were also evaluated and the findings were similar.

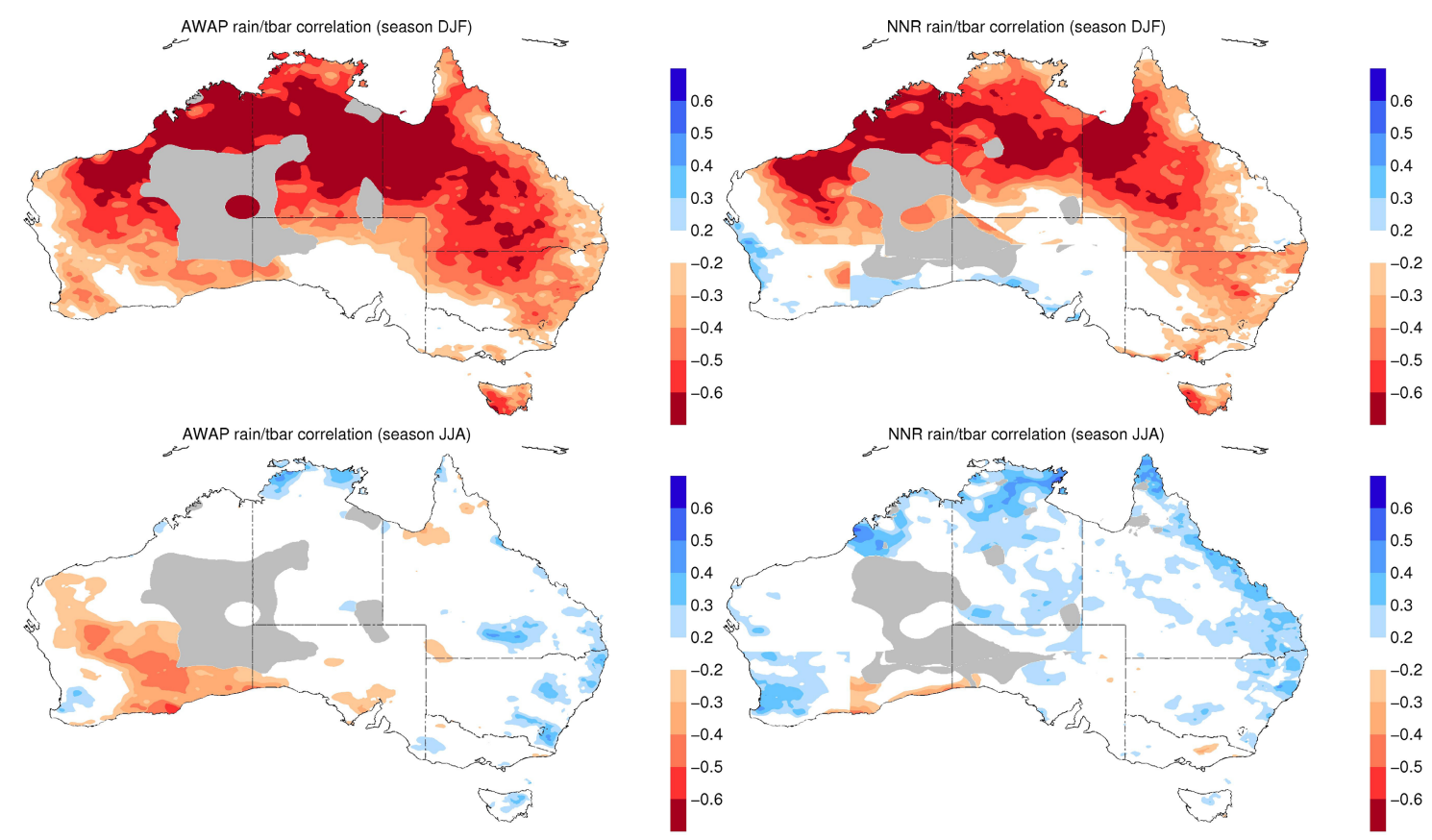

Figure 9. Maps of correlation between Rainfall and $T_{\text {mean }}$ (average of $T_{\max }$ and $T_{\min }$ ) as observed with AWAP (left column) and reproduced with the downscaled predictands (right column), for summer (upper row) and winter (lower row).

It is worth noting that, although an important step, it remains to be seen that in a future warmer world the currently observed relationship between the predictands will remain the same and that the SDM based projections will be realistic in reproducing how these relationships may evolve. This is a more complex problem to investigate and is beyond the scope of this brief communication.

\section{FUTURE PROJECTIONS}

The SDM was applied to each set of GCM predictors where these were available. In the interests of brevity, only the ensemble mean result from the $11 \mathrm{GCMs}$ are presented, but it is important to note that this methodology allows the sampling of the full range of uncertainties inherent to the climate model sensitivities.

Downscaled projections for the differences in rainfall and $\mathrm{T}_{\max }$ are compared with those from the GCMs in Figures 10 and 11 respectively. These corresponded to the difference between the A2 emissions scenario time-slice 2046-2065 and the current 1961-2000 climate.

For rainfall, in general, there is broad scale agreement between the downscaled projections and the host model projections but with sizeable local and regional differences. Most noticeable are the large-scale difference in the downscaled autumn season. Here the GCMs project an increase of rainfall across most of the continent with only a small pocket of decreased rainfall along the southern coast. The downscaled projections are more balanced showing a mixed of increases and decreases across the continent. Although a magnitude larger, the downscaled projected autumn rainfall decline along the southern coastal regions are well represented. In summer, the large-scale increases across most of the eastern side of the continent are much less pronounced in the downscaled results. However, the downscaled results suggest a sizeable increase 
in rainfall across southern Australia which is not projected by the GCMs. Note that the projected declines can be sizeable in percentage terms but small in absolute term as observed rainfall totals are small over many areas in summer. Finally, the downscaled changes of rainfall for winter (across the entire continent) and in spring (across most of the southern and eastern part) are consistent with those of the GCM-based projections. In some places (e.g. along the southern coast) the magnitude is much larger but in others its spatial extent is reduced (e.g. the south west corner of Western Australia). The projections change sign in some regions, in particular along the eastern seaboard of Australia in spring; a region where GCMs tend to perform poorly (Timbal, 2010).
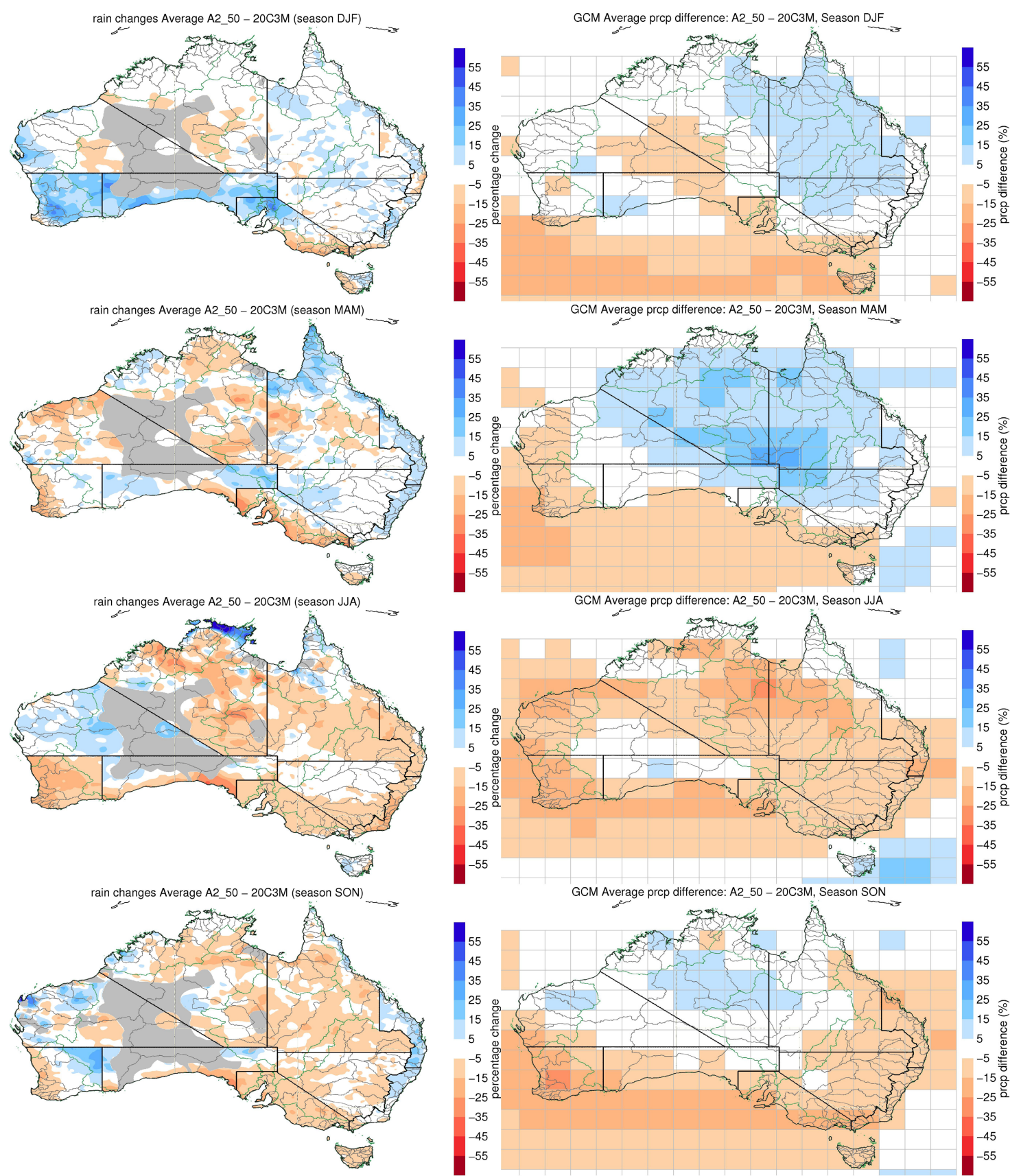

Figure 10. Maps of the differences (for each calendar season, summer to spring from top to bottom) between future projection for rainfall by 2050 using the A2 scenario and the current climate as a percentage of the current climate; maps are constructed as the ensemble mean of 11 GCMs from the CMIP3 database, either downscaled (left column) or using direct rainfall from the models interpolated to the same horizontal grid as the NNR (right column). 
A comparison of the downscaled and direct model projections for temperature (Figure 11) also reveals a general agreement with many local and regional differences. Downscaled projected warming tends to be either of similar or smaller magnitude than those given by the GCM. There are hardly any instances when the statistical downscaling of the GCMs produces a larger warming than the host models. This systematic tendency suggests a possible bias of the SDM and is indicative that the statistical linkage may not capture the full climate change signal produced by the host models. This potential underestimation of the future climate signal would be consistent with the already noted under-reproduction of the magnitude of the year to year variability (Timbal et al., 2009); that underestimation was not satisfactory reduced following the development of the inflation factor for temperature (Evans et al., 2011). The possibility that future warming is under represented by the SDM requires further investigation and is beyond the scope of this brief communication.
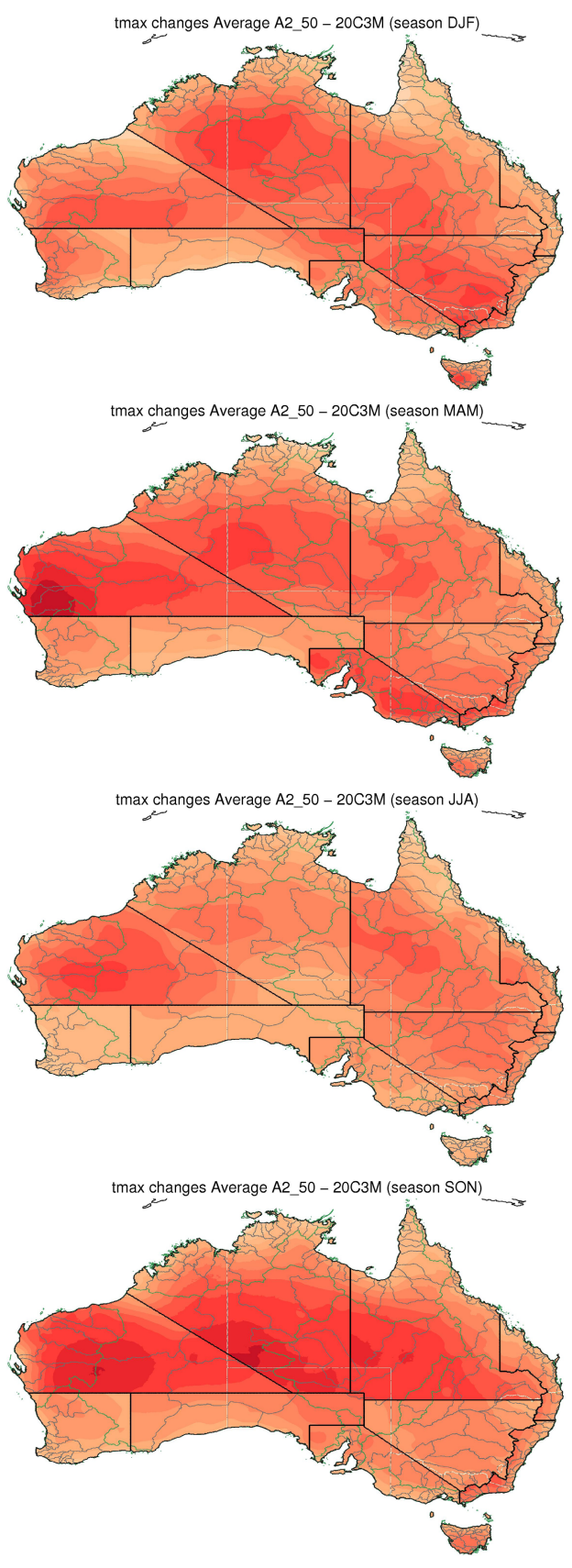

Figure 11. As per Figure 10 but for $T_{\max }$ projections
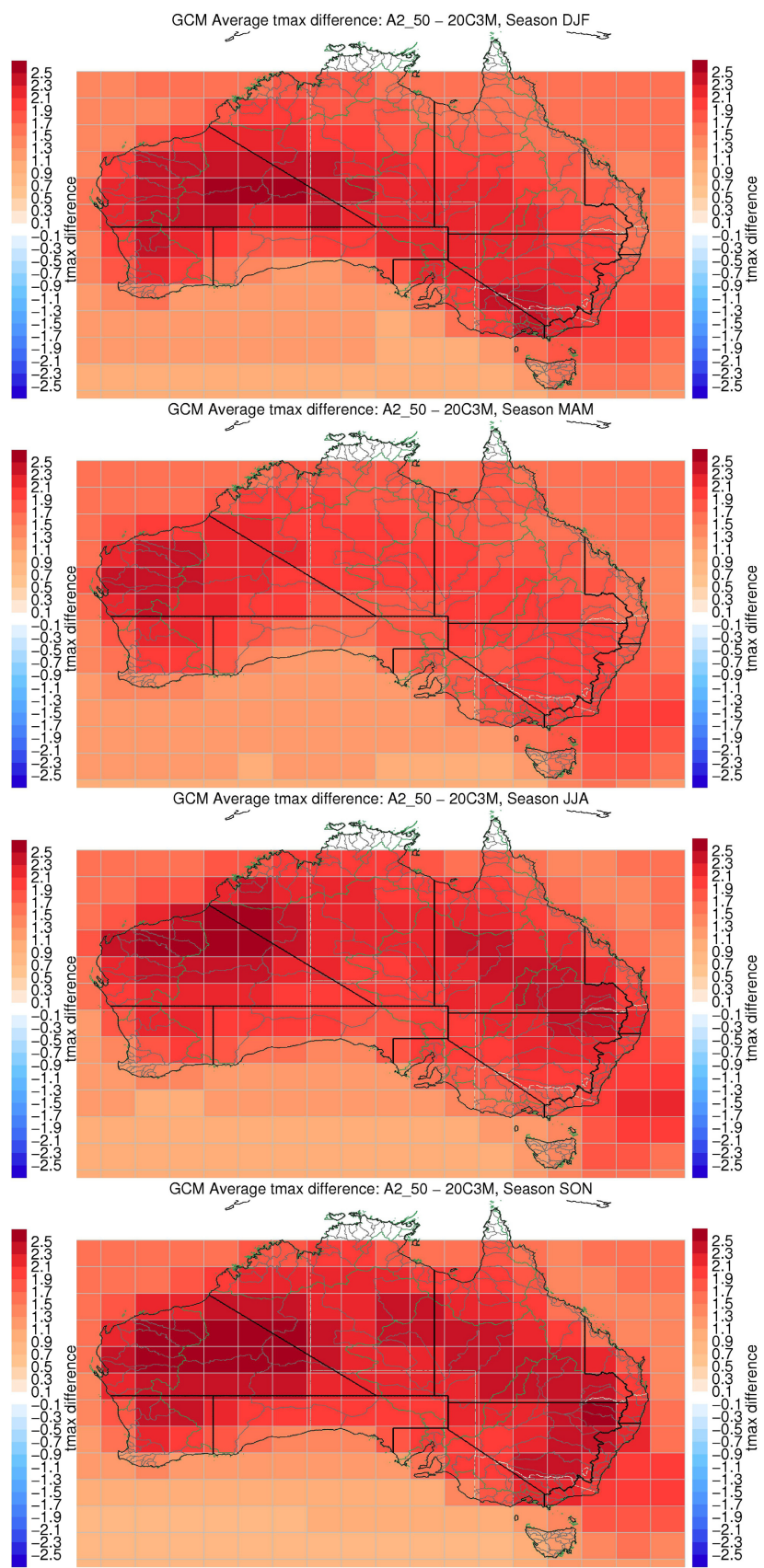


\section{DISCUSSION AND CONCLUSIONS}

We have illustrated the ability of the current SDM, to provide downscaled projections at daily time scales across Australia on a 5 by $5 \mathrm{~km}$ regular grid. It is a highly effective approach with a very low computational cost. It is flexible, adaptable and currently provides projections for time-slices in both the $21^{\text {st }}$ century for a warmer climate and for the current climate.

Currently, downscaled projections are based on the international CMIP3 database but in the near future will be applied to the CMIP5 dataset that is being assembled in time for the $5^{\text {th }}$ assessment to be delivered by the IPCC in 2014. The SDM performs as a perfect prognostic (i.e. it is not fitted to a particular GCM) and hence can be directly applied to the new CMIP5 dataset. While not challenging scientifically, it represents a tremendous task due to the size of the data involved in this exercise. The amount of daily data requested from various modelling groups as part of the CMIP5 dataset is much larger than that CMIP3 and will provide an opportunity to expand the understanding of future projections. More GCMs are likely to be available with more future emission scenarios, as well as 150 years long periods (from 1950 to 2100). In addition, shortterm climate predictions ( 20 to 30 years ahead) initialized with realistic initial conditions will also be available. While the framework to do this work is in place, the very size of the data involved makes it a lengthy and complex task. It is expected that downscaled projections are only likely to become available in stages over the course of the next two years (2012 to 2014).

The low cost of the statistical downscaling approach makes it feasible to fully evaluate the uncertainties attached to climate projections (either due to future emission pathways or due to climate model sensitivities and regional differences but not including the uncertainties attached to the downscaling approach itself). To date, high resolution projections have been made available and provided to numerous groups in Australia dealing with the risk posed by future climate change on human activities, human health or the natural environment. These downscaled projections provide researchers with the necessary input for impact modelling tools.

While this communication focuses on illustrating the ability of the current SDM when applied to gridded observations, there is room for improvement in several aspects. Some issues involve limitations of the technique in reproducing the observed variability of the local predictands. The inflation factors developed for rainfall and temperature can reduce or eliminate the impact of the underestimation of variance on both the mean and the variance of downscaled series, but do not appear to be very effective in improving the amount of inter-annual variability reproduced. This is of concern in the face of downscaled future temperature projections which are lower than the direct model projections. In addition, as noted during the evaluation of the technique using univariate approaches and confirmed using multivariate approaches, there are regions and seasons where the optimal SDM does not appear to give sufficiently good results and therefore the projections in those instances should be treated with caution. Finally, the method represents the Australian continent by a number of climate regions which are apparent as discontinuities in the results. These discontinuities have no physical basis and different future climate change on both sides of the boundary should be regarded as equally plausible. Appropriate interpolation, taking into account the uncertain nature of the projections, needs to be developed for the current crop of projections in order to provide realistic projections for applications (i.e. hydrologic catchments or others) spanning these boundaries. Currently, climate change applications requiring data across one of the existing boundaries are provided with projections based on the optimal SDM for each side of the boundary extended across the domain of interest for the particular study, in order to capture the uncertainties attached to the downscaling technique itself.

Since becoming available, dozen of research groups have accessed the downscaled gridded projections discussed here, allowing studies of the impact of future climate change on a range of human activities (e.g. wheat production in NSW, Liu et al. (2011)) or managed systems (e.g. water availability and surface run-off in catchments, Amirthahathan et al. (2011), Teng et al. (2011)). The common aim of these applications is to explore the uncertainties attached to the climate change projections in terms of the relevant local variables (e.g. wheat crop climate indices or local surface run-off).

Currently, gridded downscaled projection data are provided on an ad-hoc basis (interested readers are welcome to contact the corresponding author to access the data). However, web based tools are currently being explored to more efficiently deliver the large amount of gridded data that have been generated. 


\section{ACKNOWLEDGMENTS}

The development of the Bureau of Meteorology (BoM) statistical downscaling technique has long been supported by the Department of Climate Change and Energy Efficiency (DCCEE) as part of the Australian Climate Change Science Program (ACCSP). The predictand databases were obtained from the National Climate Centre (NCC) of the BoM. We acknowledge the international modelling groups for providing their data for analysis, the Program for Climate Model Diagnosis and Intercomparison (PCMDI) for collecting and archiving the model data, the JSC/CLIVAR Working Group on Coupled Modelling (WGCM) and their Coupled Model Intercomparison Project (CMIP) and Climate Simulation Panel for organizing the model data analysis activity, and the IPCC WG1 TSU for technical support. The IPCC Data Archive at Lawrence Livermore National Laboratory is supported by the Office of Science, U.S. Department of Energy. Thanks are due to Aurel Moise and Lawson Hanson from the BoM for transferring the CMIP3 database to Australia.

\section{REFERENCES}

Amirthahathan, G.A., M.A. Bari and B. Timbal, 2011: "Assessment of Climate change and long term water availability of Serpentine Catchment: an experimental projection”. Technical report: WIRADA, 41pp

Evans, A., B. Timbal, E. Chandler and E. Fernandez (2011): "Site-dependent variable inflation of downscaled climate series: application to the analogue method", Climate Research, submitted

Grose MR, Barnes-Keoghan I, Corney SP, White CJ, Holz GK, Bennett JB, Gaynor SM and Bindoff NL 2010, Climate Futures for Tasmania: general climate impacts technical report, Antarctic Climate \& Ecosystems Cooperative Research Centre, Hobart, Tasmania

Jones, D. A., Wang, W. and Fawcett, R. (2009): High-quality spatial climate data-sets for Australia. Australian Meteorological and Oceanographic Journal, 58 (4), 233-248.

Liu, D.L., B. Timbal and J. Mo, 2011: “A GIS-based climate change adaptation strategy tool”, International Journal of Climate Change, 3(2), 140-155

Kalnay, E., M. Kanamitsu, R. Kistler, W. Collins, D. Deaven, L. Gandin, M. Iredell, S. Saha, G. White, J. Woollen, Y. Zhu, M. Chelliah, W. Ebisuzaki, W. Higgins, J. Janowiak, K. Mo, C. Ropelewski, J. Wang, A. Leetmaa, R. Reynolds, R. Jenne and D. Joseph (1996). The NCEP/NCAR 40-year reanalysis project, Bulletin of the American Meteorological Society, 77, 437-471

Power S, Tseitkin F, Torok S, Lavery B, Dahni R, McAvaney B (1998) Australian temperature, Australian rainfall and the southern oscillation, 1910-1992: coherent variability and recent changes. Australian Meteorological Magazine 47:85-101

Solomon S., Qin, D., Manning, M., Chen, Z., Marquis, M., Averyt, K., Tignor, M. and Miller H.M. (eds.), 2007: "Climate Change 2007: The Physical Science Basis", contribution of Working Group I to the Fourth Assessment Report of the Intergovernmental Panel on Climate Change, Cambridge University Press, Cambridge, United Kingdom and New York, NY, USA, 996 pp

Teng, J., F. Chiew, B. Timbal, J. Vaze, Y. Wang, B. Wang, A. Evans, D. Kent, D. Kirono and D. Post, 2011: "Assessing historical and future runoff modelled using rainfall from the analogue downscaling method", proceedings of the MODSIM11 conference, Perth, Australia

Timbal, B., B. McAvaney, 2001: An analogue-based method to downscale surface air temperature: application for Australia, Climate Dynamics, 17, 947-963

Timbal, B., A. Dufour, B. McAvaney, 2003: An estimate of future climate change for Western France using a statistical downscaling technique, Climate Dynamics, 20, 807-823

Timbal, B., 2004: Southwest Australia past and future rainfall trends, Climate Research, 26, 233-249

Timbal, B., J. Arblaster and S. Power, (2006): Attribution of the late 20th century rainfall decline in SouthWest Australia. Journal of Climate, 19(10), 2046-2062

Timbal, B., Z. Li and E. Fernandez, (2008): The Bureau of meteorology Statistical Downscaling Model Graphical User Interface: user manual and software documentation, CAWCR research report, 4, pp 98

Timbal, B., Fernandez, E. and Li, Z, (2009): Generalization of a statistical downscaling model to provide local climate change projections for Australia. Environmental Modelling \& Software, 24 (3), 341-358

Timbal, B., 2010: "The climate of the Eastern Seaboard of Australia: a challenging entity now and for future projections", 2010 IOP Conference Series: Earth and Environmental Science 11 012013, doi: $10.1088 / 1755-1315 / 11 / 1 / 012013$

von Storch, H., 1999: On the use of "inflation" in statistical downscaling. Journal of Climate, 12, 3505-3506

Wilks, D.S., (2006), Statistical Methods in the Atmospheric Sciences, 2nd Ed. International Geophysics Series, Vol. 59, Academic Press, 627 pp. 